\title{
Restoration of enamel anti-wear properties via remineralization: Role of occlusal loading
}

\author{
Jiapin PENG, Lei LEI, Heng XIAO, Dan YANG, Jing ZHENG* ${ }^{*}$, Zhongrong ZHOU \\ Tribology Research Institute, Key Laboratory of Advanced Technologies of Materials, Ministry of Education, Southwest Jiaotong University, \\ Chengdu 610031, China
}

Received: 09 February 2021 / Revised: 05 July 2021 / Accepted: 11 October 2021

(C) The author(s) 2021.

\begin{abstract}
In this in vitro study, the restoration of acid-eroded enamel surface morphology and anti-wear properties under two conditions, mono-remineralization (treated with remineralization alone) and impact-remineralization (treated with cyclic impact followed by remineralization), are characterized to determine the effect of occlusal loading on enamel remineralization. Compared with the mono-remineralized surface, the impact-remineralized surface demonstrates better anti-wear performance, as manifested by a higher hardness and elastic modulus, as well as a lower friction coefficient and wear volume. Loading on the eroded enamel surface induces the fragmentation of hydroxyapatite nanoparticles, which aids crystal deposition and fusion during subsequent remineralization. In summary, owing to the enamel microstructure, occlusal loading can promote the restoration of enamel anti-wear properties by enhancing remineralization. Remineralization enhancement through occlusalloading-induced nanoparticle fragmentation plays a significant role in preventing human teeth from excessive wear.
\end{abstract}

Keywords: human tooth enamel; anti-wear properties; remineralization; occlusal loading; nanoparticle fragmentation

\section{Introduction}

Enamel, the outer hard shell of human teeth, has a hierarchical structure which is assembled step by step from hexagonal hydroxyapatite (HAP) nanocrystals to keyhole-like enamel rods with diameters of 5-8 $\mu \mathrm{m}$ [1]. These rods are the basic structural unit of the enamel, whereas the area between the rods (generally known as the inter-rod enamel) is rich in protein $[2,3]$. Owing to its unique microstructure, the enamel is a naturally hard biomaterial with excellent anti-wear performance [4]. Once the enamel is exposed to an acidic environment, the HAP crystals will partially dissolve, forming a rough surface with a honeycomb-like structure and a porous subsurface [5]. The structural damage caused by the acidic dissolution of HAP (known as "erosion" in dentistry [6]) decreases the hardness and elastic modulus of the enamel surface [7]. The resulting eroded enamel is more susceptible to abrasion and attrition than intact enamel [8]. In the oral environment, salivary calcium and phosphate ions induce mineral deposition on the tooth surface irregularities produced by erosion; hence, restoration of the damaged HAP crystal lattice structure can occur $[9,10]$, which is known as remineralization. The softened enamel layer caused by acid attack can be rehardened through remineralization by saliva, resulting in the restoration of enamel anti-wear properties [11, 12].

Remineralization is of high physiological importance and has been extensively investigated [13], particularly considering that the consumption of acidic beverages and foods is increasing worldwide [14, 15]. It has been widely reported that remineralizing additives can promote mineral deposition and enhance the remineralization of eroded enamel. Casein phosphopeptideamorphous calcium phosphate is believed to aid the formation of a supersaturated calcium phosphate state for maintaining high concentration gradients of

* Corresponding author: Jing ZHENG, E-mail: jzheng168@home.swjtu.edu.cn 
calcium and phosphate ions, which promote enamel remineralization and inhibit demineralization $[16,17]$. Tricalcium silicate can induce enamel remineralization by providing a stereochemical match for the oxygen atoms bonded to calcium ions and inducing the precipitation of HAP in the remineralization media [18, 19]. In 2018, Dogan et al. [20] constructed a functional biomimetic mineral layer on damaged human enamel in vitro using shADP5, a 15-AA long amelogenin-derived peptide. It was discovered that the concentration [21], viscosity [22], and $\mathrm{pH}$ [23] of the remineralization solution, as well as the remineralization time [24] affected the remineralization restoration effect. It is noteworthy that in previous studies, eroded enamel samples were only immersed in remineralization media for treatment, and no additional loading was applied [13, 25]. The mechanical and tribological properties of enamel surfaces can be improved after remineralization; however, they remain inferior to intact enamel $[26,27]$.

As an important masticatory organ in the human body, the human teeth are subjected to the external action of occlusal loading daily. In other words, even if attacked by acidic agents, the enamel still must endure the action of occlusal loading in the mouth. Studies regarding the effect of occlusal loading on the subsequent remineralization of eroded enamel are scarce; however, it has been reported that the deposition rate of HAP crystals is proportional to the specific surface area of the substrates [28]. Given the fact that the HAP nanoparticles in the enamel are split into fine particles under certain loading conditions [29, 30], it is reasonable to deduce that occlusal loading might affect the remineralization of eroded enamel and hence the restoration of anti-wear properties. Understanding the effect of occlusal loading on the remineralization and restoration of eroded enamel would aid in determining the remineralization mechanism in the mouth, and provide valuable insights into the prevention of excessive tooth wear caused by erosion.

Accordingly, occlusal loading was simulated via cyclic impact loading in the present in vitro study. The remineralization and restoration of acid-eroded enamel with and without the loading action were comparatively investigated based on a detailed characterization of the surface morphology as well as the mechanical and tribological properties. Citric acid solution was used as the erosive agent, and human saliva was used as the remineralization medium. A nano-indentation/scratch technique was employed to examine the mechanical and tribological properties of enamel. The aim of this study is to determine the effect of occlusal loading on the restoration of enamel anti-wear properties via remineralization.

\section{Materials and methods}

\subsection{Sample collection and preparation}

Fifty enamel samples were prepared from freshly extracted human third molars. All teeth, carious-free, without obvious wear, and aged between 20 and 30 years, were collected in conformity with the Chinese Psychological Society's ethical standards.

Each tooth was cut, embedded, ground, and polished to obtain a flat test surface with an average roughness $R_{\mathrm{a}}$ of less than $0.01 \mu \mathrm{m}$ using a previously reported method [8]. Less than $0.2 \mathrm{~mm}$ in height was removed by grinding and polishing for each sample. After polishing, 10 samples were treated with $5 \mathrm{~s}$ of etching using a $0.005 \mathrm{~mol} \%$ citric acid solution to remove the polishing deformation layer [29], and then used as "natural samples". Forty samples were eroded for $3 \mathrm{~min}$ via immersion in a $0.001 \mathrm{~mol} / \mathrm{L}$ citric acid solution $(\mathrm{pH}=3.20)$ with slight agitation. Among the 40 eroded samples, 10 were directly measured, 10 were treated with impact, 10 were treated with impact followed by remineralization (referred to as impact-remineralization), and the remaining 10 were treated with remineralization only (referred to as mono-remineralization). Hence, five enamel surface types were obtained: natural $(\mathrm{N})$, eroded (E), impacted (I), impact-remineralized (I-R), and mono-remineralized ( $m-R)$.

Remineralization was conducted at $37{ }^{\circ} \mathrm{C}$ by immersing the enamel samples in $5 \mathrm{~mL}$ of human saliva for $24 \mathrm{~h}$ with slight agitation. Human saliva, which was collected from 10 male donors aged 23-29 years, was mixed and centrifuged for $20 \mathrm{~min}$ at 2,000 $\mathrm{g}$ and $4{ }^{\circ} \mathrm{C}$. The supernatant of the mixture was obtained for the remineralization experiments. In previous in vitro studies, the remineralization durations were generally set to $8 \mathrm{~h}, 24 \mathrm{~h}$, as well as $28 \mathrm{~d}$ [11, 28, 31]. In the mouth, salivary calcium and phosphate ions for remineralizing enamel are continuously supplied via 
the secretion of salivary glands, and some salivary enzymes, such as alkaline phosphatase, can aid the remineralization of enamel [32-34]. During in vitro remineralization, the calcium and phosphate ions in the human saliva are gradually consumed without supply, and salivary enzymes are denatured and inactivated easily. Hence, in vitro remineralization is of lower efficiency and requires a longer duration than in vivo remineralization.

The scanning electron microscope (SEM; QUANTA200, FEI, UK) and the atomic force microscope (AFM; Cypher, Oxford Instruments Asylum Research, UK) were employed to characterize the enamel surface morphology. To determine the crystallographic features of the enamel surface, powder samples shaved from the enamel sample surfaces were examined by X-ray diffraction (XRD; X'pert pro-MPD, PANalytical, the Netherlands) and the transmission electron microscope (TEM; JEM-2100F, JEOL, Japan).

\subsection{Impact treatment}

To simulate occlusal loading in the mouth, enamel surface impact treatment was performed via cyclic loading with a frequency of $2 \mathrm{~Hz}$ at a $90^{\circ}$ inclination in a ball-on-flat configuration using a specially designed impact tester (Fig. 1). The counterpart was a silicon carbide ceramic ball with a diameter of $12 \mathrm{~mm}$. For each sample, 600 impact cycles were conducted under a normal load of $1.40 \mathrm{~N}$ at $55 \%$ relative humidity and $25{ }^{\circ} \mathrm{C}$, and the center of the impact mark, where the maximum contact pressure occurred, was used to perform subsequent tests. The associated pressure was calculated to be $314 \mathrm{MPa}$ using the mechanical parameters of sound human tooth enamel according to the Hertz contact theory [35]. In the mouth, the contact pressure applied on enamel occlusal surfaces is $121-668 \mathrm{MPa}$ during chewing $[36,37]$.

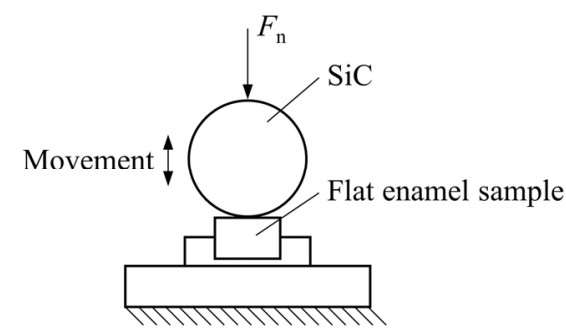

Fig. 1 Schematic diagram of impact test-rig. $F_{\mathrm{n}}$ is the normal impact force.

\subsection{Nano-indentation/scratch tests}

A nano-indenter (G200, KLA-Tencor, USA) fitted with a Berkovich diamond tip with a radius of $20 \mathrm{~nm}$ was employed to measure the mechanical properties of the enamel surfaces, including the hardness and elastic modulus. All indentation tests were conducted in a constant depth mode, and the indentation depth was set to $300 \mathrm{~nm}$. Ten indentations were created on each surface, and the space between the indentations was $100 \mu \mathrm{m}$.

A nano-scratch tester (G200, KLA-Tencor, USA) was employed to examine the tribological properties of the enamel surfaces. Unidirectional scratch tests were performed at a scratching speed of $20 \mu \mathrm{m} / \mathrm{s}$ using a conical diamond tip with a $10 \mu \mathrm{m}$ radius and a normal load applied in a constant mode $(10 \mathrm{mN})$. The length of each scratch was $100 \mu \mathrm{m}$. The lateral force during scratching was measured automatically, and a cubecorner-shaped diamond tip with a radius of $20 \mathrm{~nm}$ was used to measure the residual scratch profiles. The measured lateral force was used as friction force [38], and the coefficient of friction was obtained as the ratio of the friction force to the applied normal load. The wear loss on the enamel surface was evaluated using the scratch volume, which was calculated based on the residual scratch profile.

\subsection{Statistical analysis}

Differences in the mechanical and tribological properties between groups were analyzed via the one-way analysis of variance with Tukey's test for multiple comparisons. A $P$-value of 0.05 was set as the significance level throughout this study.

\section{Results}

Figure 2 presents the hardness and elastic modulus values of the four enamel surfaces. The eroded surface exhibits a significantly decreased hardness and elastic modulus compared with those of the natural surface $(P<0.01)$, whereas the mechanical properties of the eroded surface are improved to varying degrees by the two different remineralization treatments $(P<0.01)$. Although slightly lower than those of the natural surface, the hardness and elastic modulus of the 
(a)

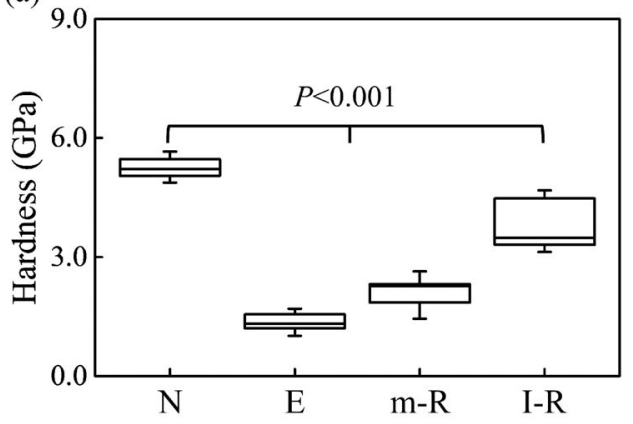

(b)

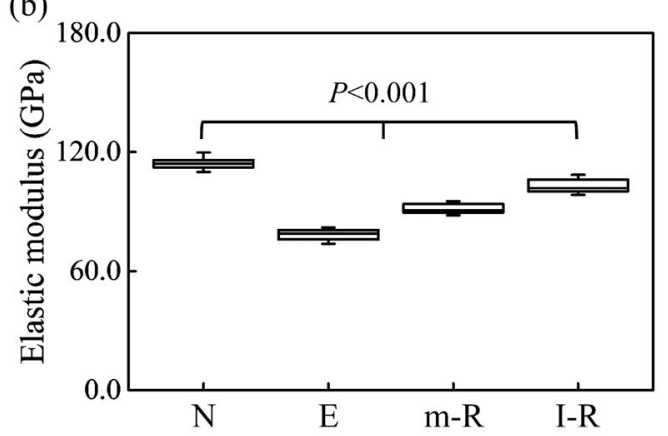

Fig. 2 Mechanical properties of four enamel surfaces: (a) hardness and (b) elastic modulus.

impact-remineralized surface are notably higher than those of the mono-remineralized surface $(P<0.01)$.

The eroded surface exhibits a considerable increase in the friction coefficient (accompanied by significant fluctuations) and wear volume compared with the natural surface $(P<0.01)$, as shown in Fig. 3 . The two remineralization treatments improve the anti-wear performance of the eroded surface $(P<0.01)$. In particular, the impact-remineralized surface indicates a significant decrease in the friction coefficient (both the mean value and fluctuation) and wear volume $(P<0.01)$.

As shown in Fig. 4, the honeycomb-like structure induced by HAP dissolution on the eroded surface is partially filled after the two remineralization treatments, particularly after impact-remineralization. Hence, the surface roughness decreases more significantly on the impact-remineralized surface than on the monoremineralized surface. However, compared with the natural surface, the two surfaces are rougher $(P<0.01)$. The deposits newly formed through remineralization in human saliva effectively reduce the increased surface porosity caused by the acid attack, as illustrated in Fig. 5. High-resolution SEM imaging shows a porosity of approximately $5.1 \%$ on the natural surface, $47.2 \%$ on the eroded surface, $14.9 \%$ on the impacted surface, $23.5 \%$ on the mono-remineralized surface, and $6.9 \%$ on the impact-remineralized surface. The effect of acid erosion on the average diameter of the HAP nanoparticles on the enamel surface is negligible $(P>0.05)$; however, the diameter distribution on the eroded surface becomes wider than that on the natural surface. Furthermore, the average particle diameter on the eroded surface decreases from 60 to $35 \mathrm{~nm}$ after 600 cycles of impact treatment at $314 \mathrm{MPa}$.
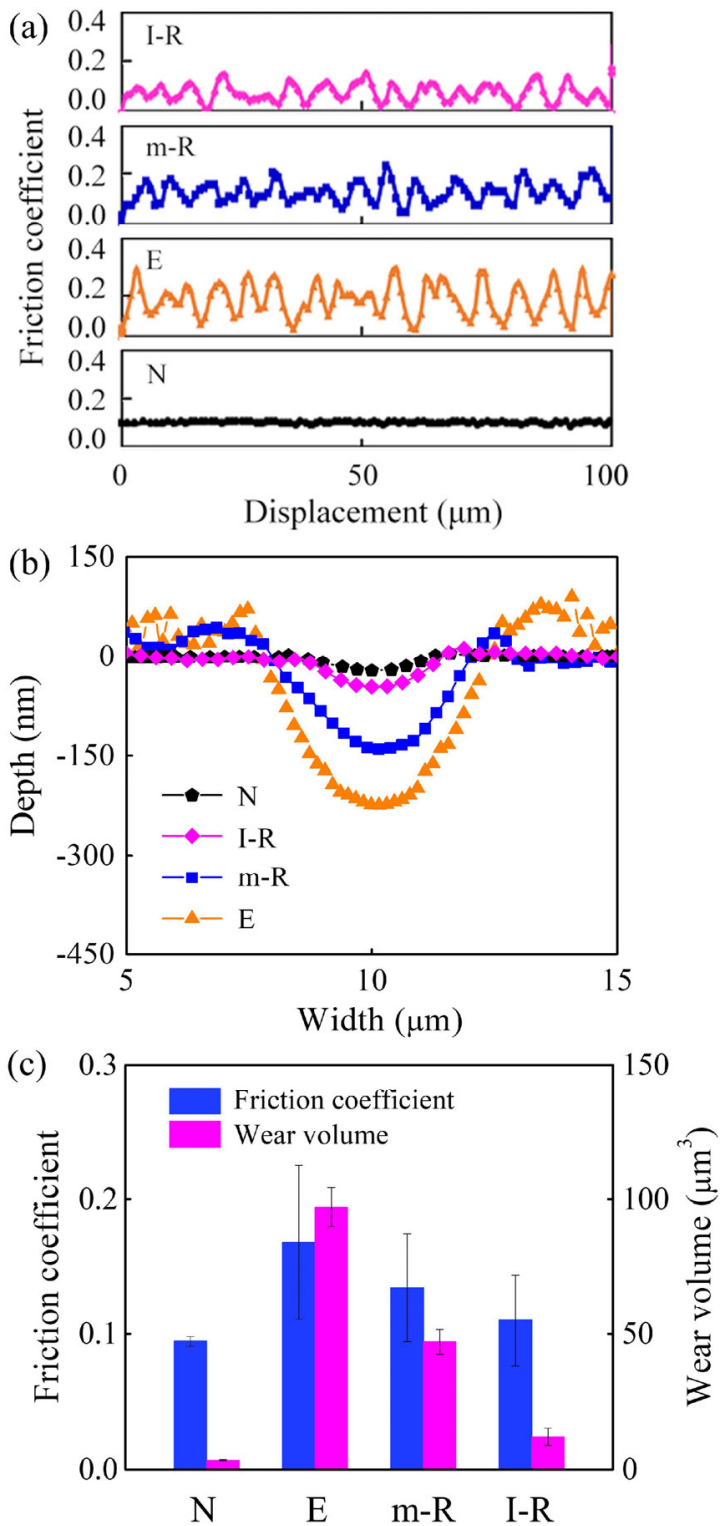

Fig. 3 Tribological performance of enamel surfaces: (a) variations of friction coefficients with displacement, (b) scratch profiles, and (c) comparison of friction coefficient and wear volume. 
(a)
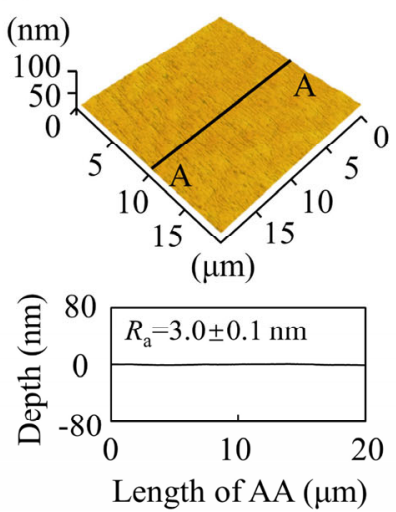

(b)
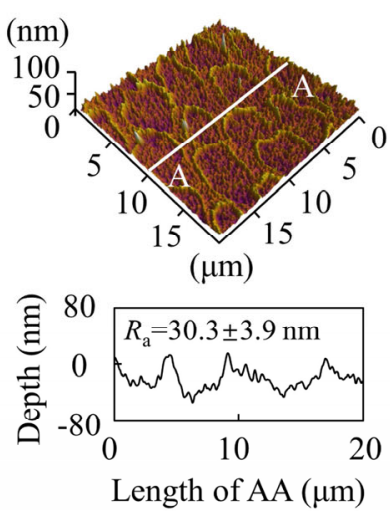

(c)
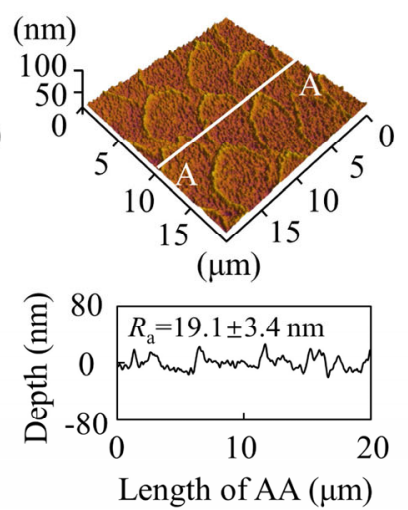

(d)
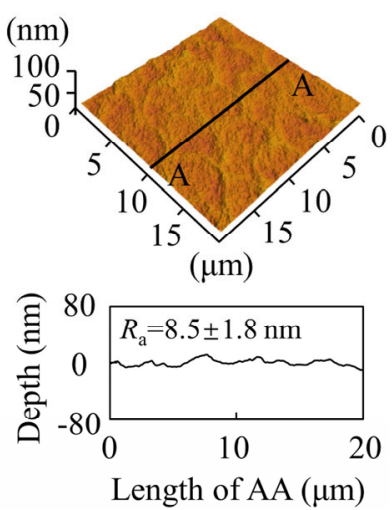

Fig. 4 AFM micrographs and profiles of (a) natural, (b) eroded, (c) mono-remineralized, and (d) impact-remineralized enamel surfaces.

(a)
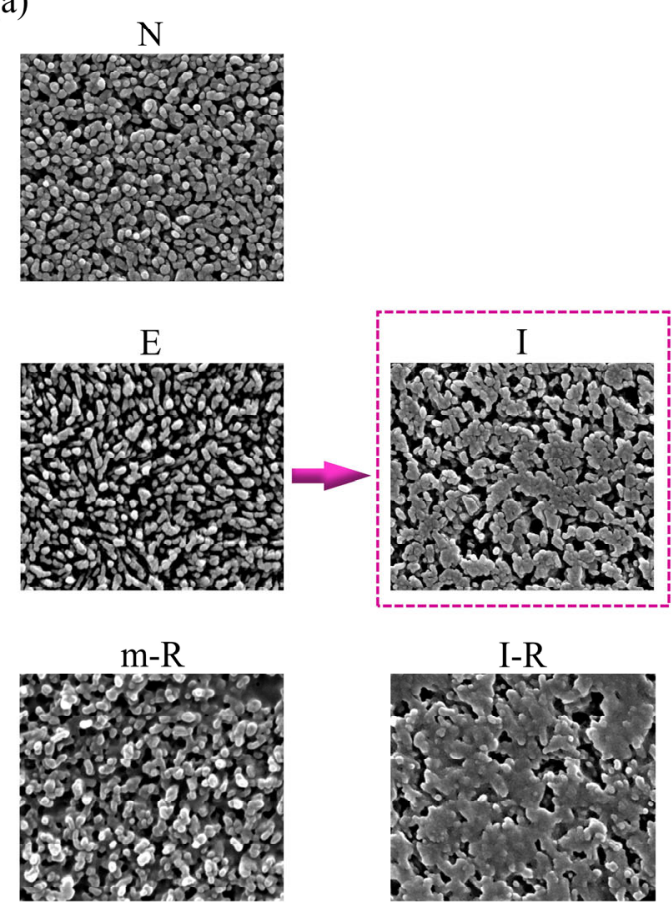

$\mathrm{I}-\mathrm{R}$

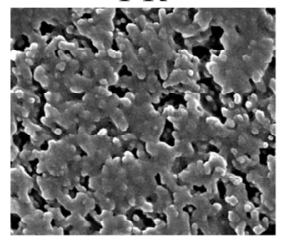

$500 \mathrm{~nm}$ (b)

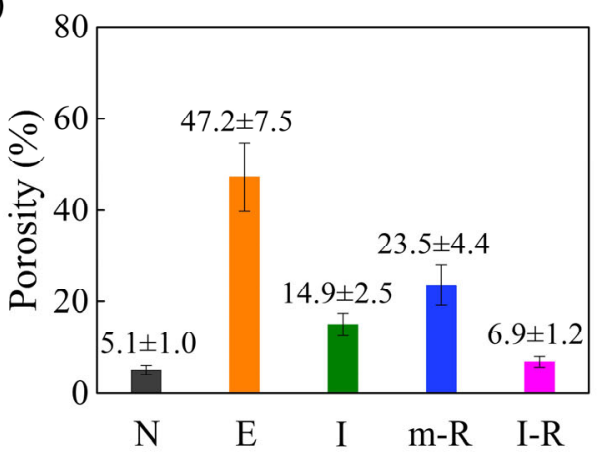

(c)

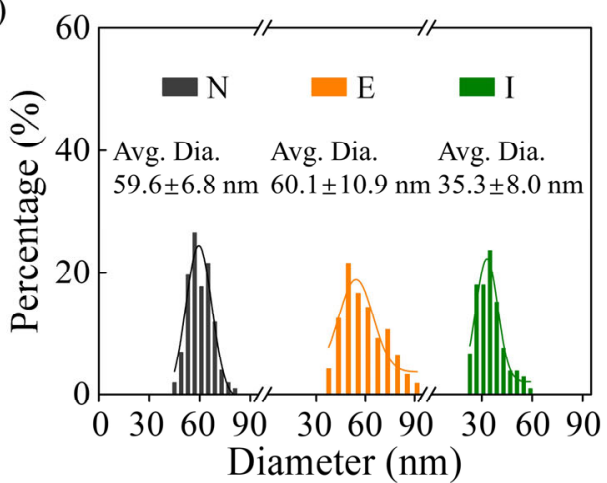

Fig. 5 SEM micrographs, porosities, and particle sizes of enamel surfaces: (a) SEM micrographs, (b) comparison of surface porosity, and (c) histograms detailing the percentages of visible nanoparticles in the accompanying images at the given diameters.

As shown in Fig. 6, the XRD patterns of the mineral powders from the five enamel surfaces match those of pure HAP. The average dimensions of the HAP crystals are calculated to be approximately $23.8,21.9$, $21.9,22.9$, and $25.3 \mathrm{~nm}$ along the $c$-axis, and 16.3, 14.3, $14.3,15.8$, and $17.7 \mathrm{~nm}$ along the $a$-axis for the natural, eroded, impacted, mono-remineralized, and impactremineralized surfaces, respectively, by applying Scherrer's formula to the XRD patterns [39].
Tapping-mode AFM images show that mineral deposition during remineralization in human saliva occurs around the HAP nanoparticles that remain on the eroded surface, which causes the voids between particles to become partially filled, as shown in Fig. 7(a). The TEM images presented in Fig. 7(b) show the morphologies of typical crystals on the four enamel surfaces and the corresponding selected area electron diffraction (SAED) patterns. The shape of the crystal on 


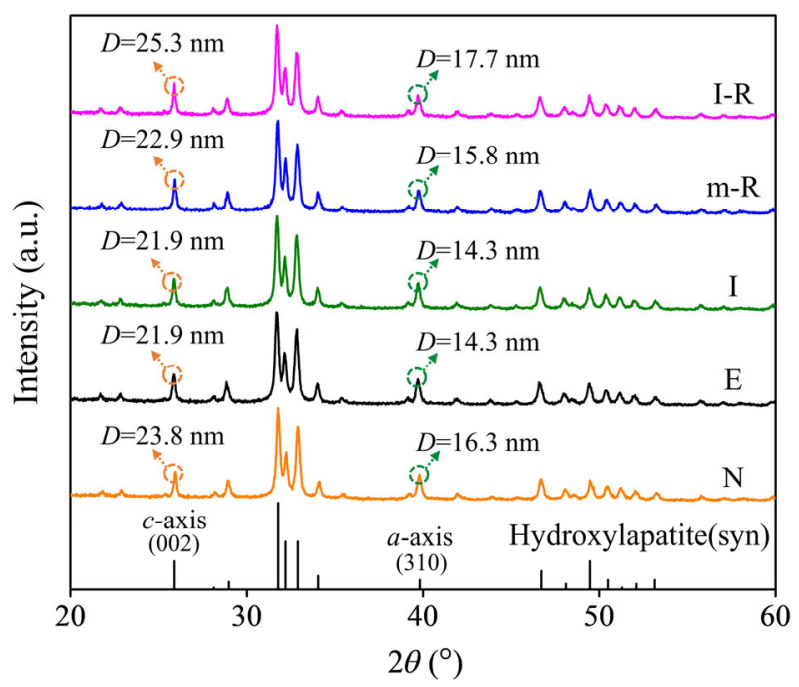

Fig. 6 XRD patterns of different enamel samples and pure HAP.

the natural surface is intact with indentation-free edges, whereas the crystal remaining on the eroded surface is irregularly shaped with sawtooth indentations on the edges due to HAP dissolution. The remaining crystal on the eroded surface is enlarged irregularly after remineralization, particularly after impactremineralization. This implies that many more fine crystals are formed and fused with the enamel crystals through impact-remineralization rather than monoremineralization. The same SAED patterns of the four samples confirm that the newly formed crystals are HAP.

(a)
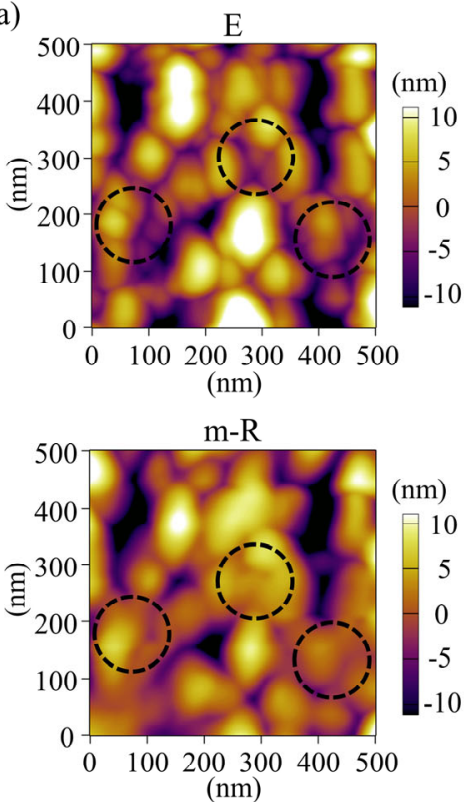

\section{Discussion}

The anti-wear performance of human teeth is vital to their physiological functions. Acid erosion is the main cause of excessive tooth wear, whereas enamel remineralization via the human saliva is an efficient way to restore dental mechanical and tribological properties [40]. The oral environment is highly complex, both chemically and in terms of loading, and is inevitably involved in enamel remineralization. Therefore, in this study, the role of occlusal loading in the remineralization and restoration of acid-eroded enamel was investigated via impact treatment. Human saliva acquired from donors was used as the remineralization medium in this study, and the remineralization duration was $24 \mathrm{~h}$. In fact, remineralization treatments for shorter durations, such as 6 and $12 \mathrm{~h}$, were conducted in our preliminary tests. It was discovered that the mechanical and tribological properties of eroded enamel exhibited a more significant restoration effect after impactremineralization compared with after monoremineralization, regardless of the remineralization duration (i.e., 6,12 , or $24 \mathrm{~h}$ ), whereas the restoration effect was positively associated with the duration of remineralization. A weak restoration effect increases the difficulty and deviation in the subsequent characterization and testing; hence, the remineralization duration was set to $24 \mathrm{~h}$. (b)
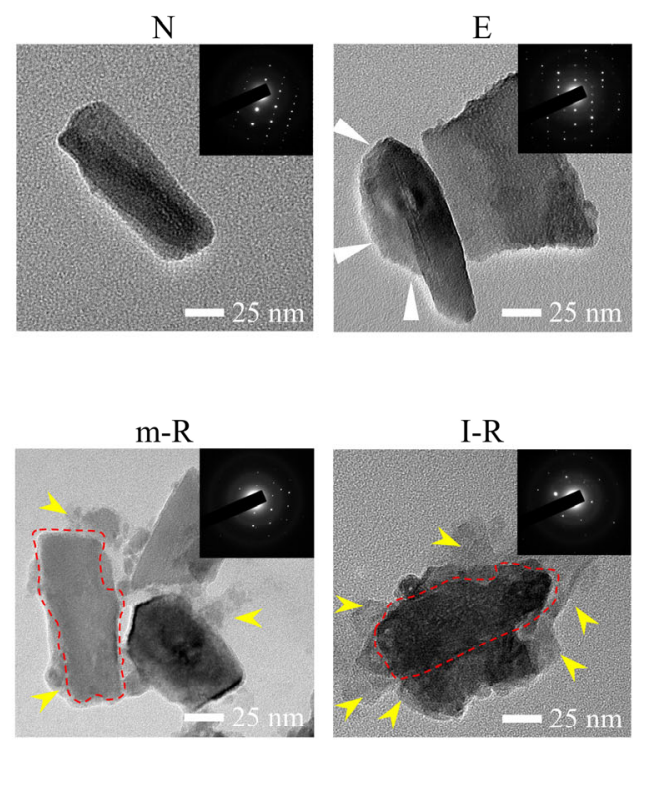

Fig. 7 AFM and TEM examination of enamel surfaces: (a) AFM images and (b) TEM morphologies and SAED patterns. 
The data presented in Figs. 2 and 3 show that, compared with the natural surface, the enhancements to the eroded surface's mechanical and tribological properties are limited after mono-remineralization, but significant after impact-remineralization. The erosive lesion of the enamel surface results from HAP dissolution, which led to a direct enamel crystal loss and to undissolved enamel crystals with perforations or defects in their centers and defects of various sizes on their lateral surfaces [41]. A decrease in the crystal size of the eroded surface confirms the occurrence of these defects (Fig. 6). The remaining crystals are sparsely arranged and form a softened layer on the eroded surface [41, 42]. In situ AFM imaging using a $10 \mathrm{~nm}$ radius silicon tip demonstrates that the remaining enamel crystals in the softened layer provided sites for the deposition of newly formed HAP crystals during remineralization (Fig. 7(a)), which is consistent with previous results [10]. The new crystals fuse with each other or with the remaining enamel crystals, thereby restoring the defects of the latter (see the red profiles in Fig. 7(b)) and filling the voids caused by HAP dissolution (see the dotted black circles in Fig. 7(a)). As shown in Figs. 5(a) and 7(b), more fine crystals and more significant crystal fusion occur around the remaining enamel crystals after impact-remineralization compared with after mono-remineralization. The HAP nanoparticle lattice on the eroded enamel surface did not deform as easily under the external action of impact loading; as such, the sizes and shapes of the individual crystallites on the eroded surface remain almost unchanged after 600 impact treatment cycles at $314 \mathrm{MPa}$ (Fig. 6). Hence, it can be inferred that impact loading on the eroded surface aided the deposition and fusion of new fine HAP crystals on the lateral surface of the remaining enamel crystals during subsequent remineralization in human saliva. This inference is supported by the XRD results for enamel powders from different surfaces. As shown in Fig. 6, the HAP crystal size on the eroded surface increases slightly after the two remineralization treatments, particularly after impact-remineralization. The deposition and fusion of HAP crystals contributed to the densification and rehardening of the eroded enamel surface, which effectively decreased the friction coefficient (both the mean value and fluctuation) and wear loss of the eroded enamel. Hence, the mechanical and tribological properties of the enamel impaired by erosion were improved more significantly by impact-remineralization than by mono-remineralization. Hence, it is evident that impact loading plays a role in promoting the remineralization of eroded enamel.

In this study, the friction and wear behavior of enamel samples under the specified scratch testing conditions was dominated by ploughing effect. As shown in Fig. 8, the scratch tip is pressed into the enamel to a certain depth under the action of a normal load and then slid forward, thereby causing material removal on the enamel surface, that is, enamel wear. For the ploughing wear, the friction force can be calculated as Eq. (1) [43]:

$$
F_{\mathrm{f}}=S \sigma_{\mathrm{s}}=\frac{1}{2} d h \sigma_{\mathrm{s}}=h^{2} \sigma_{\mathrm{s}} \tan \theta
$$

where $F_{\mathrm{f}}$ is the friction force, $S$ is the projected area of the tip-enamel contact interface on the vertical plane, $\sigma_{\mathrm{s}}$ is the yield strength of the enamel, $h$ is the pressing depth of the tip into the enamel surface, $\theta$ is the semicone angle of the tip, and $d$ is equal to $2 h \tan \theta$. The pressing depth is negatively associated with the enamel surface hardness and elastic modulus, whereas the yield strength is positively associated with the surface hardness [44]. As shown in Eq. (1), the effect of the pressing depth on the friction force is more significant than that of the yield strength. Hence, the friction force during scratching is negatively associated with the enamel surface hardness and elastic modulus. Under the same normal loading level, the greater the pressing depth of the tip into the enamel surface is, the more significant the material removal on the enamel surface. As shown in Fig. 2, the surface hardness and elastic modulus of the four enamel samples are ranked as follows: $\mathrm{E}<\mathrm{m}-\mathrm{R}<\mathrm{I}-\mathrm{R}<\mathrm{N}$. Hence, the friction force (friction coefficient) and wear loss of the eroded enamel
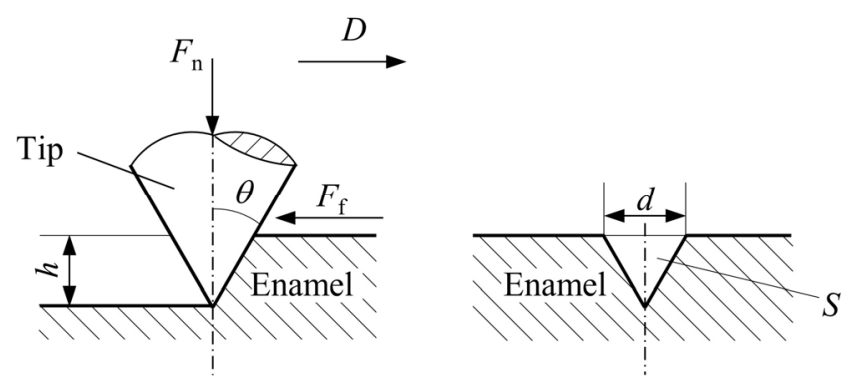

Fig. 8 Schematic diagram of ploughing wear induced by scratch tip on enamel surface. 
surface decreased after the two remineralization treatments, particularly after impact-remineralization.

The role of impact loading in enamel remineralization appears to be associated with its effect on the enamel surface microstructure, as shown in Fig. 5. Enamel rods are the structural units of the human tooth enamel, each of which is composed of orderly arranged nanofibers assembled by HAP crystals [45]. The nanofibers, also known as nanoparticles, are uniform in size, with an average diameter of approximately $60 \mathrm{~nm}$ [46]. The dissolution of HAP caused by acid attack not only reduces the size of the nanoparticles, but also causes them to lean by increasing the gaps between them. As shown in Fig. 5(a), randomly distributed transverse nanofibers appear on the eroded surface, which confirms the leaning of HAP nanoparticles induced by acid erosion. Hence, although their average diameter (approximately $60 \mathrm{~nm}$ ) did not change significantly, the nanoparticles on the eroded surface differed in size, causing a wider diameter distribution. Additionally, the surface porosity is as high as $47.2 \%$ owing to the increased number of voids between particles, and it is significantly higher than that of the natural surface (approximately 5.1\%). After 600 cycles of impact treatment at $314 \mathrm{MPa}$, the average nanoparticle size decreases to $35 \mathrm{~nm}$ on the eroded surface, accompanied by a narrow diameter distribution and a decrease in the surface porosity to $14.9 \%$. It is evident that impact loading on the eroded surface induces the fragmentation of HAP nanoparticles and hence decreases the surface porosity. In other words, impact loading on the eroded surface barely affects the size or shape of the individual crystallites, but can change the enamel surface microstructure by inducing HAP nanoparticle fragmentation.

As discussed above, the enamel crystals remaining on the eroded surface are crucial in providing sites for the deposition of new crystals during remineralization. The nanoparticle fragmentation induced by impact loading causes more crystals to be exposed on the eroded enamel surface, thereby providing more sites for crystal deposition. In addition, nanoparticle fragmentation results in decreased porosity on the eroded surface, thereby benefiting the fusion of newly formed crystals. The impact-remineralized surface porosity is approximately $6.9 \%$, which is markedly lower than that of the mono-remineralized surface (approximately 23.5\%) and similar to that of the natural surface (approximately 5.1\%) (Fig. 5(b)), thereby confirming that effective crystal deposition and fusion occurred in the softened layer. Therefore, it can be inferred that impact loading promotes the remineralization potential of the eroded enamel surface in human saliva by inducing nanoparticle fragmentation. This inference is in fact supported by the results of previous studies. It has been reported that the remineralization restoration effect of enamel is negatively associated with the extent of enamel erosion because a higher erosion extent corresponds to fewer remaining enamel crystals and a higher porosity in the softened layer [31].

HAP crystals with a diameter of approximately $20 \mathrm{~nm}$ are the fundamental units of the enamel. They are glued together via proteins, and further assemble into fibrous nanoparticles measuring $60-70 \mathrm{~nm}$ in diameter [1]. Once the surface of the enamel is subjected to a contact pressure equivalent to the bonding strength between the crystals, the fibrous nanoparticles risk splitting into fine particles [4]. Recently, we discovered that under normal loading, the fibrous nanoparticles on a natural enamel surface did not split into fine particles until a contact pressure of $730 \mathrm{MPa}[29,30]$. In other words, the bonding strength between the crystals was approximately $730 \mathrm{MPa}$. In the present study, the contact pressure applied to the eroded enamel surface was only $314 \mathrm{MPa}$; nonetheless, HAP nanoparticle fragmentation occurred, suggesting that the critical stress to induce nanoparticle fragmentation is reduced on eroded surfaces. This is primarily due to changes in the enamel surface microstructure caused by acid attack. First, the eroded surface becomes extremely rough owing to the acidic dissolution of HAP (Fig. 4), which causes an increased surface stress concentration under the external action of impact loading. Second, the HAP nanoparticles in the softened layer are composed of the remaining undissolved crystals, which are filled with defects of various sizes and exhibit an impaired bonding strength with each other. Third, owing to the dissolution of HAP during erosion, some remaining nanoparticles on the eroded surface lean or tip over, and hence easily split into fine particles under impact loading [29]. Therefore, it is clear that erosion enhances nanoparticle fragmentation on the enamel surface.

The enamel, which is the outer shield of the human 
teeth, is exposed to the oral environment and undergoes occlusal loading daily [47]. The contact stress generated in the enamel during normal chewing ranges from 121 to $668 \mathrm{MPa}[36,37]$. This implies that nanoparticle fragmentation barely occurs on the sound enamel surface under normal occlusal loading. However, as discussed above, erosion enhances nanoparticle fragmentation on the enamel surface via the dissolution of HAP. Hence, for the eroded enamel surface, normal occlusal loading in the mouth can fragment the HAP particles remaining in the softened layer. It is noteworthy that in the mouth, the teeth will impact each other under the action of occlusal loading, and micro-sliding will occur. It has been reported that micro-sliding under a certain normal load can induce HAP nanoparticle fragmentation $[4,30]$, and the critical normal load on the enamel surface to induce HAP nanoparticle fragmentation is lower under micro-sliding than under impacting [30]. Therefore, it can be deduced that in the oral environment, the action of occlusal loading on the eroded enamel surface during impacting and micro-sliding induces HAP nanoparticle fragmentation, which promotes surface remineralization via the human saliva by increasing the number of sites for HAP crystal deposition.

Figure 9 presents the enamel microstructure and the role of occlusal loading in enamel remineralization. The fragmentation of HAP nanoparticles depends significantly on the bonding strength between the internal fine crystal particles [30, 48]. Erosion results in crystal defects through HAP dissolution, and the defects increase with the extent of erosion [41]. The more obvious the crystal defects, the weaker the bonding between them. This implies that in the oral environment, owing to the action of occlusal loading, the eroded enamel surface contains adequate sites for crystal deposition provided that the erosion is not excessive. Clearly, occlusal loading enhances enamel remineralization by inducing HAP nanoparticle fragmentation; hence, erosive lesions of the enamel surface caused by the daily human diet, particularly the impaired mechanical and tribological properties, can be effectively restored through remineralization via the human saliva. The fragmentation of HAP nanoparticles under the action of occlusal loading is attributed to the hierarchical microstructure of the enamel [29, 30]. Hence, the remineralization enhancement induced by occlusal loading is derived from the unique microstructure of the enamel. Namely, it is a hierarchical microstructure that enables the enamel to effectively restore the antiwear properties damaged by acid erosion through remineralization in the oral environment, thereby preventing human teeth from excessive wear.

As a self-restoration process, remineralization is critical for maintaining the anti-wear performance of the enamel. The restoration of the mechanical and

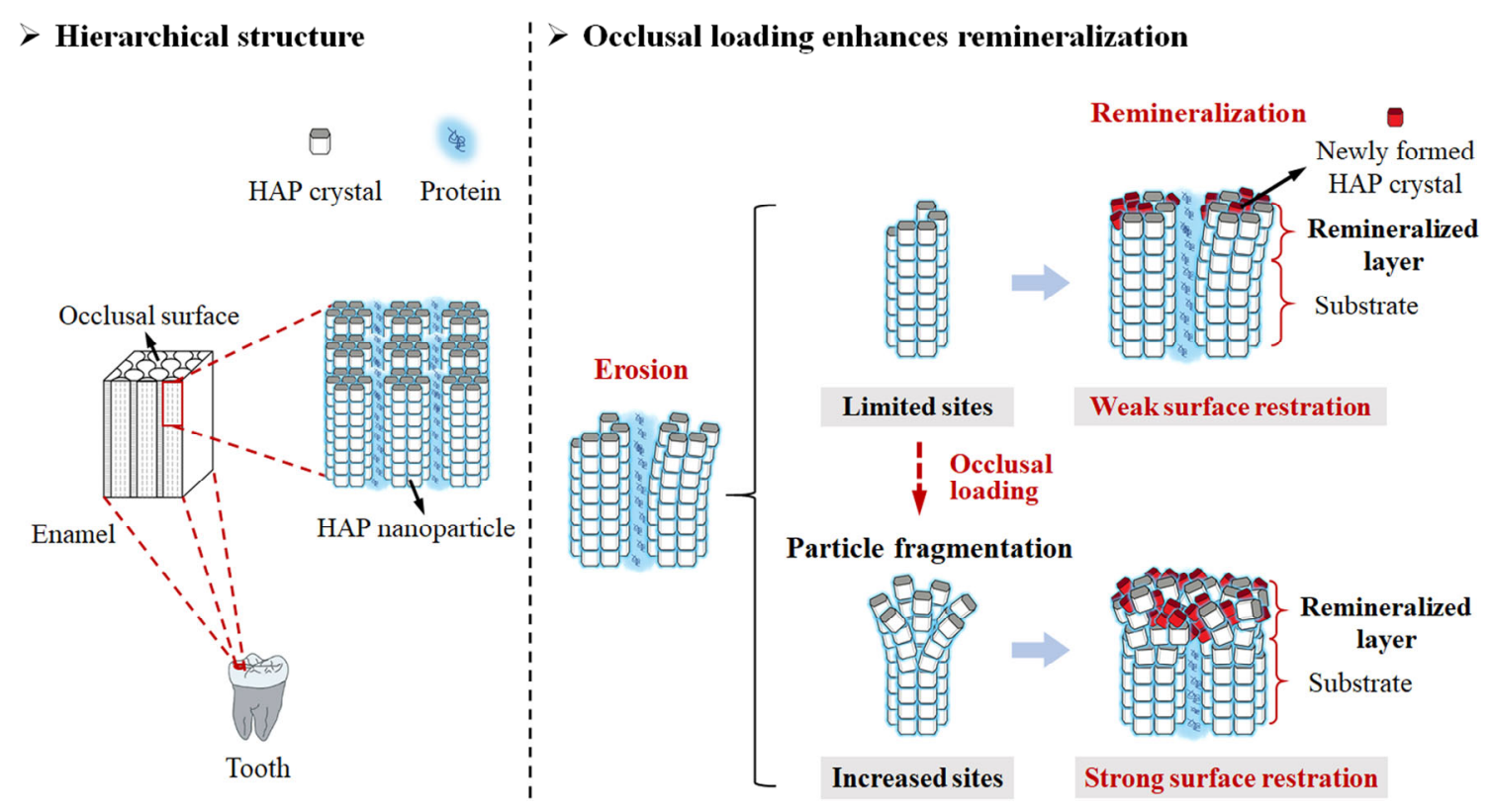

Fig. 9 Schematic diagrams of enamel microstructure and the role of occlusal loading in enamel remineralization. 
tribological properties of acid-eroded enamel via remineralization has been investigated widely $[9,49$, 50]. However, occlusal loading was not considered in previous studies, and the results showed that remineralization restoration induced by the human saliva was unsatisfactory without the use of additives $[12,26]$. The results obtained in this study show that owing to the unique hierarchical microstructure of the enamel, occlusal loading can enhance enamel remineralization in the oral environment by inducing HAP nanoparticle fragmentation and increasing the number of sites for the deposition of newly formed HAP crystals, thereby promoting the restoration of anti-wear properties. The restoration of eroded enamel depends significantly on the remaining enamel crystals in the softened layer. These findings extend the understanding pertaining to the restoration of enamel remineralization in the oral environment and the dental anti-wear mechanism. The occlusal-loading-enhanced remineralization mechanism allows the human teeth to tolerate a certain degree of acid attack. In other words, moderate consumption of acidic foods/beverages will not deteriorate the health of the human teeth. Acid attacks of long durations and/or the low $\mathrm{pH}$ value (or high acid concentration) on a sound enamel surface, as well as the severe mechanical friction on an eroded enamel surface reduce the remaining enamel crystals in the softened layer [5, 7, 8, 51]. Hence, consuming low-pH foods or drinks continuously and drinking acidic beverages such as soft drinks when eating tough foods are not recommended. Additionally, the occlusal-loading-enhanced remineralization behavior of eroded enamel suggests that the unique microstructure of the enamel endows the material with a certain self-adaption ability to undergo various service conditions such as an acidic oral environment. This provides valuable insights into the development of new dental materials. The detailed effect of occlusal loading on the growth of HAP crystals formed through remineralization in the human saliva will be investigated in future studies.

\section{Conclusions}

The effect of occlusal loading on the remineralization and anti-wear restoration of eroded enamel was investigated in vitro. Based on the experimental conditions and results, the following conclusions are obtained.

1) Compared with a mono-remineralized surface, an impact-remineralized surface exhibits better anti-wear performance, as manifested by a higher hardness and elastic modulus, as well as a lower friction coefficient and wear volume.

2) Loading on the eroded enamel surface increases the number of sites for crystal deposition by inducing the fragmentation of HAP nanoparticles in the softened layer, thereby enhancing enamel remineralization and promoting the restoration of anti-wear properties.

3) The remineralization and restoration of the enamel enhanced by occlusal loading, which is essential for preventing excessive tooth wear, is attributed to the unique hierarchical microstructure of enamel. Restoration depends significantly on the remaining enamel crystals in the softened layer on the eroded tooth surface; hence, for the daily management of teeth health, consuming low-pH foods or drinks continuously and drinking acidic beverages when eating tough foods are not recommended.

\section{Acknowledgements}

This work was supported by the National Natural Science Foundation of China (Nos. 52035001, 51675449, and 51535010) and the 111 Project of China (No. B20008).

Open Access This article is licensed under a Creative Commons Attribution 4.0 International License, which permits use, sharing, adaptation, distribution and reproduction in any medium or format, as long as you give appropriate credit to the original author(s) and the source, provide a link to the Creative Commons licence, and indicate if changes were made.

The images or other third party material in this article are included in the article's Creative Commons licence, unless indicated otherwise in a credit line to the material. If material is not included in the article's Creative Commons licence and your intended use is not permitted by statutory regulation or exceeds the permitted use, you will need to obtain permission directly from the copyright holder.

To view a copy of this licence, visit http://creativecommons.org/licenses/by/4.0/. 


\section{References}

[1] Cui F Z, Ge J. New observations of the hierarchical structure of human enamel, from nanoscale to microscale. $J$ Tissue Eng Regen Med 1(3): 185-191 (2007)

[2] Ge J, Cui F Z, Wang X M, Feng H L. Property variations in the prism and the organic sheath within enamel by nanoindentation. Biomaterials 26(16): 3333-3339 (2005)

[3] Lawn B R, Lee J J W, Chai H. Teeth: Among nature's most durable biocomposites. Annu Rev Mater Res 40(1): 55-75 (2010)

[4] Zheng J, Li Y, Shi M Y, Zhang Y F, Qian L M, Zhou Z R. Microtribological behaviour of human tooth enamel and artificial hydroxyapatite. Tribol Int 63: 177-185 (2013)

[5] Cheng Z J, Wang X M, Cui F Z, Ge J, Yan J X. The enamel softening and loss during early erosion studied by AFM, SEM and nanoindentation. Biomed Mater 4(1): 015020 (2009)

[6] Schlueter N, Amaechi B T, Bartlett D, Buzalaf M A R, Carvalho T S, Ganss C, Hara A T, Huysmans M C D N J M, Lussi A, Moazzez R, et al. Terminology of erosive tooth wear: Consensus report of a workshop organized by the ORCA and the cariology research group of the IADR. Caries Res 54(1): 2-6 (2020)

[7] Zheng J, Xiao F, Qian L M, Zhou Z R. Erosion behavior of human tooth enamel in citric acid solution. Tribol Int 42(11-12): 1558-1564 (2009)

[8] Zheng J, Huang H, Shi M Y, Zheng L, Qian L M, Zhou Z R. In vitro study on the wear behaviour of human tooth enamel in citric acid solution. Wear 271(9-10): 2313-2321 (2011)

[9] Arsecularatne J A, Hoffman M J. The wear behaviour of remineralised human dental enamel: An in vitro study. Wear 444-445: 203165 (2020)

[10] Yanagisawa T, Miake Y. High-resolution electron microscopy of enamel-crystal demineralization and remineralization in carious lesions. J Electron Microsc 52(6): 605-613 (2003)

[11] Amaechi B T, Higham S M. In vitro remineralisation of eroded enamel lesions by saliva. J Dent 29(5): 371-376 (2001)

[12] Arsecularatne J A, Hoffman M. An in vitro study of the microstructure, composition and nanoindentation mechanical properties of remineralizing human dental enamel. $J$ Phys $D$ : Appl Phys 47(31): 315403 (2014)

[13] Cochrane N J, Cai F, Huq N L, Burrow M F, Reynolds E C. New approaches to enhanced remineralization of tooth enamel. $J$ Dent Res 89(11): 1187-1197 (2010)

[14] Hooper S M, Newcombe R G, Faller R, Eversole S, Addy $\mathrm{M}$, West N X. The protective effects of toothpaste against erosion by orange juice: Studies in situ and in vitro. J Dent 35(6): 476-481 (2007)

[15] Tahmassebi J F, Duggal M S, Malik-Kotru G, Curzon M E
J. Soft drinks and dental health: A review of the current literature. J Dent 34(1): 2-11 (2006)

[16] Poggio C, Lombardini M, Dagna A, Chiesa M, Bianchi S. Protective effect on enamel demineralization of a CPP-ACP paste: An AFM in vitro study. Journal of Dentistry 37(12): 949-954 (2009)

[17] Ekambaram M, Mohd Said S N B, Yiu C K Y. A review of enamel remineralisation potential of calcium- and phosphate-based remineralisation systems. Oral Health Prev Dent 15(5): 415-420 (2017)

[18] Dong Z, Chang J, Joiner A, Sun Y. Tricalcium silicate induces enamel remineralization in human saliva. J Dent Sci 8(4): 440-443 (2013)

[19] Sahai N, Anseau M. Cyclic silicate active site and stereochemical match for apatite nucleation on pseudowollastonite bioceramic-bone interfaces. Biomaterials 26(29): 5763-5770 (2005)

[20] Dogan S, Fong H, Yucesoy D T, Cousin T, Gresswell C, Dag S, Huang G, Sarikaya M. Biomimetic tooth repair: Amelogenin-derived peptide enables in vitro remineralization of human enamel. ACS Biomater Sci Eng 4(5): 1788-1796 (2018)

[21] Chung H Y, Huang K C. Effects of peptide concentration on remineralization of eroded enamel. $J$ Mech Behav Biomed Mater 28: 213-221 (2013)

[22] Vissink A, Gravenmade E J, Gelhard T B, Panders A K, Franken M H. Rehardening properties of mucin- or CMCcontaining saliva substitutes on softened human enamel. Caries Res 19(3): 212-218 (1985)

[23] Arnold W H, Haase A, Hacklaender J, Gintner Z, Bánóczy J, Gaengler P. Effect of $\mathrm{pH}$ of amine fluoride containing toothpastes on enamel remineralization in vitro. BMC Oral Health 7(1): 14 (2007)

[24] Lechner B D, Röper S, Messerschmidt J, Blume A, Magerle R. Monitoring demineralization and subsequent remineralization of human teeth at the dentin-enamel junction with atomic force microscopy. ACS Appl Mater Interfaces 7(34): 18937-18943 (2015)

[25] Zheng L, Peng J, Zheng J, Liu D, Zhou Z. Surface properties of eroded human primary and permanent enamel and the possible remineralization influence of CPP-ACP. Wear 376-377: 251-258 (2017)

[26] Zheng L, Zheng J, Zhang Y F, Qian L M, Zhou Z R. Effect of CPP-ACP on the remineralization of acid-eroded human tooth enamel: Nanomechanical properties and microtribological behaviour study. J Phys D: Appl Phys 46(40): 404006 (2013)

[27] Zheng L, Zheng J, Weng L Q, Qian L M, Zhou Z R. Effect of remineralization on the nanomechanical properties and microtribological behaviour of acid-eroded human tooth enamel. Wear 271(9-10): 2297-2304 (2011) 
[28] Cate J M, Arends J. Remineralization of artificial enamel lesions in vitro. Caries Res 11(5): 277-286 (1977)

[29] Peng J, Xiao H, Yang D, Lei L, Zheng J, Zhou Z. Surface hardening behavior of enamel by masticatory loading: Occurrence mechanism and antiwear effect. ACS Biomater Sci Eng 6(8): 4454-4461 (2020)

[30] Xia J, Tian Z R, Hua L, Chen L, Zhou Z, Qian L, Ungar P S. Enamel crystallite strength and wear: Nanoscale responses of teeth to chewing loads. $J R$ Soc Interface 14(135): 20170456 (2017)

[31] Collys K, Cleymaet R, Coomans D, Michotte Y, Slop D. Rehardening of surface softened and surface etched enamel in vitro and by intraoral exposure. Caries Res 27(1): 15-20 (1993)

[32] Larsen M J, Jensen A F, Madsen D M, Pearce E I F. Individual variations of $\mathrm{pH}$, buffer capacity, and concentrations of calcium and phosphate in unstimulated whole saliva. Arch Oral Biol 44(2): 111-117 (1999)

[33] Kim E E, Wyckoff H W. Reaction mechanism of alkaline phosphatase based on crystal structures: Two-metal ion catalysis. J Mol Biol 218(2): 449-464 (1991)

[34] Kaufman E, Lamster I B. Analysis of saliva for periodontal diagnosis-a review. J Clin Periodontol 27(7): 453-465 (2000)

[35] Johnson K L. Contact Mechanics. London (UK): Cambridge University Press, 1985.

[36] He L H, Swain M V. Understanding the mechanical behaviour of human enamel from its structural and compositional characteristics. J Mech Behav Biomed Mater 1(1): 18-29 (2008)

[37] Gibbs C H, Mahan P E, Lundeen H C, Brehnan K, Walsh E $\mathrm{K}$, Holbrook W B. Occlusal forces during chewing and swallowing as measured by sound transmission. J Prosthet Dent 46(4): 443-449 (1981)

[38] Mendas M, Benayoun S. Comparative study of abrasion via microindentation and microscratch tests of reinforced and unreinforced lamellar cast iron. Friction 7(5): 457-465 (2019)

[39] Scherrer P. Bestimmung der inneren Struktur und der Größe von Kolloidteilchen mittels Röntgenstrahlen. In Kolloidchemie Ein Lehrbuch. Berlin: Springer, 1912: 387-409. (in German)

[40] Buzalaf M A R, Hannas A R, Kato M T. Saliva and dental erosion. J Appl Oral Sci 20(5): 493-502 (2012)

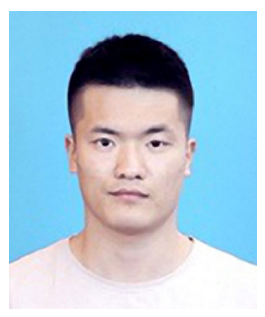

Jiapin PENG. He received his B.S. degree from Jiangsu University, Zhenjiang, 2015. He is now a Ph.D. candidate at the Tribology Research
[41] Hisako T, Takaaki Y, Noriyuki T, Shosaburo T. Growth and fusion of apatite crystals in the remineralized enamel. Journal of Electron Microscopy 39(4): 238-244 (1990)

[42] DeRocher K A, Smeets P J M, Goodge B H, Zachman M J, Balachandran P V, Stegbauer L, Cohen M J, Gordon L M, Rondinelli J M, Kourkoutis L F, et al. Chemical gradients in human enamel crystallites. Nature 583(7814): 66-71 (2020)

[43] Wen S, Huang P. Sliding friction and its applications. In Principles of Tribology. Wen S, Huang P, Eds. Singapore: John Wiley \& Sons Singapore Pte. Ltd., 2017: 225-251.

[44] Cahoon J R, Broughton W H, Kutzak A R. The determination of yield strength from hardness measurements. Metall Trans 2(7): 1979-1983 (1971)

[45] Bechtle S, Ang S F, Schneider G A. On the mechanical properties of hierarchically structured biological materials. Biomaterials 31(25): 6378-6385 (2010)

[46] White S N, Luo W, Paine M L, Fong H, Sarikaya M, Snead M L. Biological organization of hydroxyapatite crystallites into a fibrous continuum toughens and controls anisotropy in human enamel. J Dent Res 80(1): 321-326 (2001)

[47] Elfallah H M, Bertassoni L E, Charadram N, Rathsam C, Swain M V. Effect of tooth bleaching agents on protein content and mechanical properties of dental enamel. Acta Biomater 20: 120-128 (2015)

[48] Zheng S Y, Zheng J, Gao S S, Yu B J, Yu H Y, Qian L M, Zhou Z R. Investigation on the microtribological behaviour of human tooth enamel by nanoscratch. Wear 271(9-10): 2290-2296 (2011)

[49] Wang Y L, Chang H H, Chiang Y C, Lin C H, Lin C P. Strontium ion can significantly decrease enamel demineralization and prevent the enamel surface hardness loss in acidic environment. J Formos Med Assoc 118(1): 39-49 (2019)

[50] Austin R S, Giusca C L, Macaulay G, Moazzez R, Bartlett D W. Confocal laser scanning microscopy and area-scale analysis used to quantify enamel surface textural changes from citric acid demineralization and salivary remineralization in vitro. Dent Mater 32(2): 278-284 (2016)

[51] Larsen M, Nyvad B. Enamel erosion by some soft drinks and orange juices relative to their $\mathrm{pH}$, buffering effect and contents of calcium phosphate. Caries Res 33(1): 81-87 (1999)

Institute at Southwest Jiaotong University, Chengdu, China. His research interests include the tribology of natural teeth and bionic tribology. 
Jing ZHENG. She received her Ph.D. degree in mechanical engineering in 2005 from Southwest Jiaotong University, Chengdu, China. She joined the Tribology Research
Institute at Southwest Jiaotong University in 2000. Her current position is a professor. Her research areas cover the tribology of natural teeth and dental materials, bio-lubrication, and bionic tribology. 